\section{A METHOD FOR CHARACTERIZING COPLANAR WAVEGUIDE-TO- MICROSTRIP TRANSITIONS, AND ITS APPLICATION TO THE MEASUREMENT OF MICROSTRIP DEVICES WITH COPLANAR MICROPROBES}

M. C. Maya, A. Lázaro, P. DePaco, and L. Pradell Universitat Politècnica de Catalunya (UPC) Dept. TSC Campus Nord UPC, Mòdul D3

08034 Barcelona, Spain

\section{Received 10 May 2003}

ABSTRACT: This paper presents a method for characterizing coplanar waveguide-to-microstrip ( $C P W-M)$ transitions by using on-wafer coplanar $(C P W)$ microprobes. It is based on extracting the transmission matrix of the $C P W-M$ transition from the measurement of the $\mathrm{S}$ parameters of two microstrip transmission lines of different lengths, each of which includes two CPW-M transitions. To verify the proposed method, the $\mathrm{S}$ parameters of a zero-length line, which is composed of two CPW-M transitions ("thru" connection) and has not been used in the transition characterization, are measured up to $40 \mathrm{GHz}$ and are compared to those extracted and obtained from electromagnetic simulations. The method is applied to the measurement of a microstrip coupled-line filter embedded in CPW-M transitions. (C) 2003 Wiley Periodicals, Inc. Microwave Opt Technol Lett 39: 373-378, 2003; Published online in Wiley InterScience (www.interscience.wiley.com). DOI 10.1002/mop. 11222

Key words: coplanar waveguide-to-microstrip transition; network analyzer calibration; electromagnetic simulation; de-embedding

\section{INTRODUCTION}

The fast development of MMIC technology and its integration in subsystems at microwave and millimeter-wave frequencies requires the flexibility to use a combination of planar technologies, for example, microstrip and coplanar waveguide (CPW). An example of a joint use of CPW and microstrip transmission media is the need for accurate measurements of microstrip devices at microwave frequencies, which can be accomplished by testing the microstrip device embedded in CPWs, through coplanar (on-wafer) microprobes in a wafer-probe station. To this end, CPW-tomicrostrip (CPW-M) transitions have been developed in research laboratories [1-3] and commercially (for example, the Coplanarto-Microstrip Test Interface Circuit from JMICRO ${ }^{\mathrm{TM}}$ Technology), as illustrated by the configuration for transmission measurements shown in Figure 1, in which a microstrip line is connected to two CPM-M transitions.

On-wafer measurements of CPW devices are performed by using well-known network analyzer calibration techniques [4-8] with appropriate on-wafer CPW reflection ("short," "open," and "matched load") and transmission (a number of CPW lines with different lengths) standards. On-wafer CPW standards are usually well known and good enough to be used up to frequencies exceeding $100 \mathrm{GHz}$, such as the ISS ${ }^{\mathrm{TM}}$ substrate from Cascade-Microtech, which results in a very accurate calibration at millimeterwaves. In a similar way, the measurement of microstrip devices through on-wafer microprobes can also be done by applying one of the former on-wafer calibration procedures to microstrip calibration standards (compatible with the CPW-M transitions), in order to directly refer the measurements to the microstrip plane [see planes $1^{\prime}-2$ ' in Fig. 1(a)]. However, microstrip reflection standards are not ideal and/or difficult to be modeled at millimeter-wave

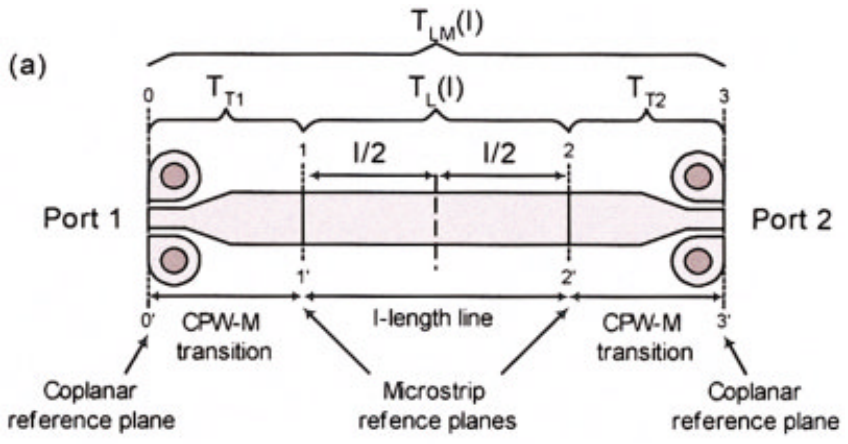

(b)

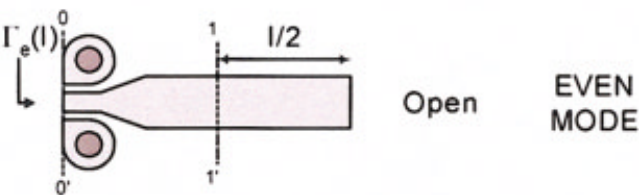

(c)

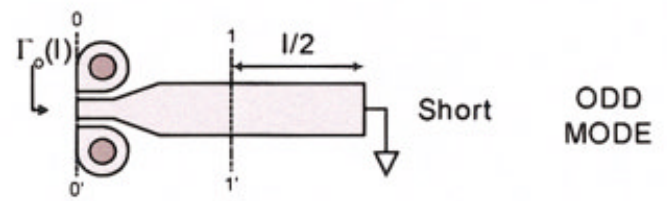

Figure 1 (a) Microstrip transmission line with two symmetric CPW-M transitions; (b) even-mode analysis; (c) odd-mode analysis [Color figure can be viewed in the online issue, which is available at www.interscience. wiley.com.]

frequencies ("short" includes via-holes, and "open" features radiation loss). As an example (see Fig. 2), the measured return loss $\left(\left|S_{11}\right|\right.$ expressed in $\mathrm{dB}$ ) at $50 \mathrm{GHz}$ of a single JMICRO ${ }^{\mathrm{TM}} \mathrm{CPW}-\mathrm{M}$ open-ended transition ("open" standard), is 1-dB higher than insertion losses of a "thru" standard, composed by a double CPW-M transition (CPW-M-CPW), and 0.5-dB higher than a short-ended transition ("short" standard), due to the losses associated to the reflection standards.

Alternatively, on-wafer standards can be used for calibration at the CPW (on-wafer) planes [ $0^{\prime}$ and $3^{\prime}$ in Fig. 1(a)], and then the reference plane can be shifted to the microstrip plane [planes $1^{\prime}-2^{\prime}$ in Fig. 1(a)] by taking into account the CPW-M transition effects. To this end, in [9] a method for measuring transmission parameters of microstrip devices through on-wafer microprobes, which does not use reflection standards, but uses two nonreflecting lines only, is presented. Other authors propose circuit models for the CPW-M transition which are fit to measurements [10], or to electromagnetic simulations [11]. Methods for the measurement of characteristic impedances of CPW and microstrip lines in the millimeter-wave range have also been proposed [12-13]. These methods are based on extracting the ABCD-matrix of CPW-to-CPW transitions [12], or the $\mathrm{ABCD}$ matrix of a circuit model associated to differences between two probe-tip-to-CPW line junctions and to CPW-M transitions [13].

In this paper, the method of characterizing CPW-to-CPW transitions in [12] is generalized, applied to the extraction of CPW-M transitions, and compared to electromagnetic simulations. The transmission-matrix ( $T$-matrix) of the CPW-M transition is extracted from the measurement of the $S$ parameters of two microstrip transmission lines of different lengths, which include two CPW-M transitions, using on-wafer probes. No restriction in the ratio between the line lengths is made, in contrast with [12], where one line length should be twice that of the other. However, differ- 

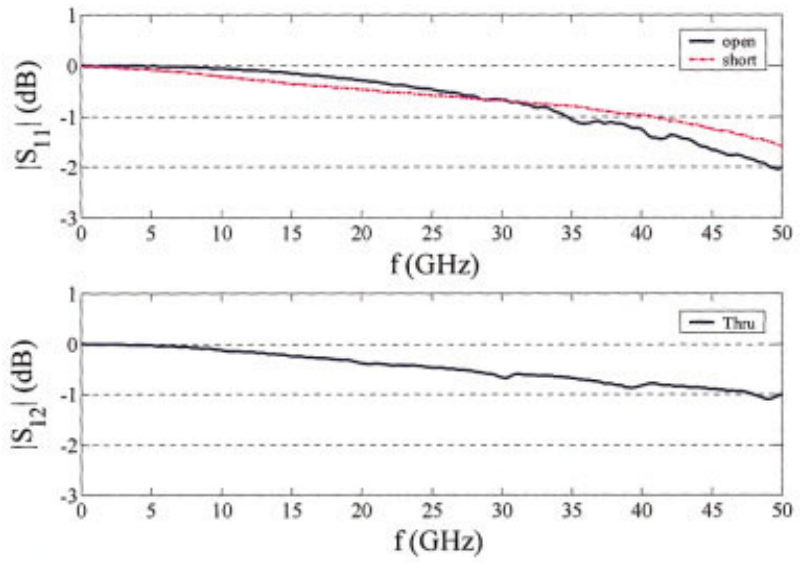

Figure 2 Reflection coefficient of "short" and "open" standards and transmission coefficient of "thru" standard from JMICRO"T Technology. [Color figure can be viewed in the online issue, which is available at www.interscience.wiley.com.]

ences of a half wavelength (and their multiples) between line lengths are not allowed. The on-wafer calibration is performed with on-wafer CPW standards using the LRRM technique [8]. Experimental verification of the extraction of a CPW-M JMICRO ${ }^{\mathrm{TM}}$ transition up to $40 \mathrm{GHz}$ is presented and its results are used for measuring the $S$ parameters of a microstrip coupled line filter.

\section{CPW-TO-MICROSTRIP TRANSITION CHARACTERIZATION}

Figure 1(a) shows the microwave CPW-M transition geometry which will be considered in this paper. It is based on a well-

$$
T_{L}(l)=\left[\begin{array}{cc}
\cosh (\gamma \boldsymbol{l})-\left(\frac{Z^{2}+Z_{0}^{2}}{2 Z Z_{0}}\right) \cdot \sinh (\gamma \boldsymbol{l}) & \left(\frac{Z^{2}-Z_{0}^{2}}{2 Z Z_{0}}\right) \cdot \sinh (\gamma l) \\
-\left(\frac{Z^{2}-Z_{0}^{2}}{2 Z Z_{0}}\right) \cdot \sinh (\gamma l) & \cosh (\gamma l)+\left(\frac{Z^{2}+Z_{0}^{2}}{2 Z Z_{0}}\right) \cdot \sinh (\gamma l)
\end{array}\right],
$$

where $Z_{0}$ is the reference impedance (usually, $Z_{0}=50 \Omega$ ). In contrast to [9], where the line impedance is $Z_{0}$, an arbitrary impedance $Z$, is assumed here.

Using the same procedure as in the TRL calibration [4-5], the propagation constant $\gamma$ can be obtained by combining the measurement of two lines: line $A$ with length $l_{A}$, and line $B$ with length $l_{B}$. Then, applying Eq. (1) to each line, the following expression is derived:

$$
T_{T 1} \cdot T_{L}\left(l_{B}\right) \cdot T_{L}\left(l_{A}\right)^{-1} \cdot T_{T 1}^{-1}=T_{L M}\left(l_{B}\right) \cdot T_{L M}\left(l_{A}\right)^{-1} .
$$

Eq. (3) express a similarity transformation $\left(A \cdot K \cdot A^{-1}=M\right)$ between matrices $K=T_{L}\left(l_{B}\right) \cdot T_{L}\left(l_{A}\right)^{-1}$ and $M=T_{L M}\left(l_{B}\right) \cdot$ $T_{L M}\left(l_{A}\right)^{-1}$, with two mathematical properties: (i) trace conservation $(\operatorname{trace}(K)=\operatorname{trace}(M))$, and (ii) determinant conservation $(\operatorname{det}(K)=\operatorname{det}(M))$ [8]. Then, substituting (2) into (3) and using the trace conservation property, $\cosh \left(\gamma \boldsymbol{l}_{T}\right)$ can be determined from

$$
\cosh \left(\gamma l_{T}\right)=\frac{1}{2} \cdot \operatorname{trace}\left(T_{L M}\left(l_{B}\right) \cdot T_{L M}\left(l_{A}\right)^{-1}\right),
$$

matched line fabricated on a low-loss substrate with two via-holes, at the CPW port, for achieving good contact between the CPW (on-wafer) microprobe ground tips and the substrate ground plane. The adapter from JMICRO ${ }^{\mathrm{TM}}$ Technology, used throughout this paper, is an example of commercially available transitions with the former design principle.

To characterize the CPW-M transition, a mathematical method similar to those applied for correcting the systematic errors in vector network analyzers (auto-calibration techniques [7-8]) is applied. It is based on measuring transmission standards only, as in $[9,12,13]$, which contain the CPW-M transitions to be characterized. The principle of error correction is developed from the measurements of two transmission lines with different lengths, as follows. Figure 1(a) shows the layout of a line of length $l$. We denote the transmission-matrices ( $T$ matrices) of the CPW-M transition at ports 1 and 2 as $T_{T 1}$ and $T_{T 2}$, respectively, and the $T$ matrix of the microstrip line of length $l$ as $T_{L}(l)$. The measuredline $T$ matrix $T_{L M}$ can be expressed as

$$
T_{L M}(\boldsymbol{l})=T_{T 1} \cdot T_{L}(\boldsymbol{l}) \cdot T_{T 2},
$$

where the $T_{L M}$ matrix is computed from the measured line's $S$ parameters. Suppose that the ground-to-ground spacing (substrate thickness) is kept small, in order to assume quasi-TEM wave propagation in the line, from transmission line theory, a section of a transmission line, with length $l$, having a complex propagation constant $\gamma$ and complex characteristic impedance $Z$, has the following $T$ matrix:

where $l_{T}$ is the difference between the lengths of line $A$ and $B$ $\left(l_{T}=l_{B}-l_{A}\right)$. In contrast to [12], where the line-length ratio is restricted, that is, the length of one of the lines must be twice the other one, here no restriction in the ratio between the line lengths is made, with the exception of differences of a half wavelength (and their multiples) between line lengths, which are not allowed.

To compute the line characteristic impedance, we apply the determinant conservation in Eq. (3); by expressing the determinant of $T_{L}\left(l_{B}\right) \cdot T_{L}\left(l_{A}\right)^{-1}$, and also $\cosh \left(\gamma l_{T}\right)$ [Eq. (4)], in terms of the $S$ parameters, $S_{I T i j}(i=1,2$ and $j=1,2)$ of a line with length $l_{T}$ and the same impedance characteristic $Z$, the following expression for $S_{I T 21}$ is found [12]:

$$
S_{l_{T 21}}=\frac{b \pm \sqrt{b^{2}-4 a}}{2 a}
$$

with

$$
a=\operatorname{det}\left(T_{L M}\left(l_{B}\right) \cdot T_{L M}\left(l_{A}\right)^{-1}\right), \quad b=2 \cosh \left(\gamma l_{T}\right),
$$


where the term $S_{I T 11} S_{l T 22}$ has been neglected because the CPW tapered line has been designed to match both the geometrical shape and impedance of the microstrip transmission line and the CPW probe. Knowing $S_{I T 21}$, we can derive the microstrip line characteristic impedance $Z$ as follows:

$$
Z=\frac{-b_{1} \pm \sqrt{b_{1}^{2}-4 Z_{0}^{2}}}{2}
$$

where

$$
b_{1}=\frac{2 Z_{0}\left(S_{l_{T 21}} \cosh \left(\gamma l_{T}\right)-1\right)}{S_{l_{T 21}} \sinh \left(\gamma l_{T}\right)} .
$$

The sign ambiguity in Eqs. (5) and (7) is easily resolved, since a correct determination of both signs will result in a positive value in the real part of $Z$.

Next, assuming that the two transitions are identical, but one of them is rotated $180^{\circ}$, the lines with the transitions have a symmetry plane (see Fig. 1); therefore, an even/odd mode analysis can be performed to obtain the $T$ matrices of the CPW-M transitions, given by

$$
T_{T 1}=\left[\begin{array}{cc}
T_{T 11} & T_{T 12} \\
T_{T 21} & T_{T 22}
\end{array}\right] ; \quad T_{T 2}=\left[\begin{array}{cc}
T_{T 11} & -T_{T 21} \\
-T_{T 12} & T_{T 22}
\end{array}\right],
$$

where the determinant of the matrix $\Delta=T_{T 11} \cdot T_{T 22}-T_{T 12} \cdot$ $T_{T 21}=1$, since the transitions are reciprocal. For each line measured (with a length 1 ), we can split the measured $S$ parameters as [see Fig. 1(b) and 1(c)]:

$$
S(l)=\frac{1}{2}\left[\begin{array}{ll}
\Gamma_{e}(l)+\Gamma_{o}(l) & \Gamma_{e}(l)-\Gamma_{o}(l) \\
\Gamma_{e}(l)-\Gamma_{o}(l) & \Gamma_{e}(l)+\Gamma_{o}(l)
\end{array}\right],
$$

where, in Eq. (10) only, the input reflection coefficient for each case (even and odd mode) can be expressed as:

$$
\begin{gathered}
\Gamma_{e}(\boldsymbol{l})=\frac{Z_{I N}^{e}(\boldsymbol{l})-Z_{0}}{Z_{I N}^{e}(\boldsymbol{l})+Z_{0}}=S_{11}(\boldsymbol{l})+S_{12}(\boldsymbol{l}), \\
\Gamma_{o}(\boldsymbol{l})=\frac{Z_{I N}^{o}(\boldsymbol{l})-Z_{0}}{Z_{I N}^{o}(\boldsymbol{l})+Z_{0}}=S_{11}(\boldsymbol{l})-S_{12}(\boldsymbol{l}) .
\end{gathered}
$$

The input impedance for each mode is expanded as

$$
\begin{gathered}
\frac{Z_{I N}^{e}(l)}{Z_{0}}=\frac{\frac{T_{1}}{T_{3}} \cdot\left(Z_{L}^{e}+Z_{0}\right)+\frac{T_{2}}{T_{3}} \cdot\left(Z_{L}^{e}-Z_{0}\right)}{Z_{L}^{e}+Z_{0}+\frac{T_{4}}{T_{3}} \cdot\left(Z_{L}^{e}-Z_{0}\right)}, \\
\frac{Z_{I N}^{o}(l)}{Z_{0}}=\frac{\frac{T_{1}}{T_{3}} \cdot\left(Z_{L}^{o}+Z_{0}\right)+\frac{T_{2}}{T_{3}} \cdot\left(Z_{L}^{o}-Z_{0}\right)}{Z_{L}^{o}+Z_{0}+\frac{T_{4}}{T_{3}} \cdot\left(Z_{L}^{o}-Z_{0}\right)},
\end{gathered}
$$

where $T_{1}, T_{2}, T_{3}$, and $T_{4}$ are defined as a function of the transition $T$ parameters:

$$
\begin{aligned}
& T_{1}=T_{T 22}+T_{T 12}, \\
& T_{2}=T_{T 21}+T_{T 11},
\end{aligned}
$$

$$
\begin{aligned}
& T_{3}=T_{T 22}-T_{T 12}, \\
& T_{4}=T_{T 21}-T_{T 11},
\end{aligned}
$$

and $Z_{L}^{e}=Z \cdot \operatorname{coth}(\gamma l / 2)$ and $Z_{L}^{o}=Z \cdot \tanh (\gamma l / 2)$ are the input impedance of an open and shorted transmission line of length $1 / 2$, respectively, $Z$ is the impedance computed from Eq. (7), and $\gamma$ is computed from Eq. (4). Using Eqs. (13) and (14) for each line ( $A$ and $B)$, the following equation system can be found:

$$
\begin{array}{r}
\left(Z_{L A}^{e}+Z_{0}\right) \cdot \frac{T_{1}}{T_{3}}+\left(Z_{L A}^{e}-Z_{0}\right) \cdot \frac{T_{2}}{T_{3}}-\frac{Z_{I N}^{e}\left(l_{A}\right)}{Z_{0}}\left(Z_{L A}^{e}-Z_{0}\right) \cdot \frac{T_{4}}{T_{3}} \\
=\frac{Z_{I N}^{e}\left(l_{A}\right)}{Z_{0}}\left(Z_{L A}^{e}+Z_{0}\right), \\
\left(Z_{L A}^{o}+Z_{0}\right) \cdot \frac{T_{1}}{T_{3}}+\left(Z_{L A}^{o}-Z_{0}\right) \cdot \frac{T_{2}}{T_{3}}-\frac{Z_{I N}^{o}\left(l_{A}\right)}{Z_{0}}\left(Z_{L A}^{o}-Z_{0}\right) \cdot \frac{T_{4}}{T_{3}} \\
=\frac{Z_{I N}^{o}\left(l_{A}\right)}{Z_{0}}\left(Z_{L A}^{o}+Z_{0}\right),
\end{array}
$$

$$
\begin{aligned}
\left(Z_{L B}^{e}+Z_{0}\right) \cdot \frac{T_{1}}{T_{3}}+\left(Z_{L B}^{e}-Z_{0}\right) \cdot \frac{T_{2}}{T_{3}}-\frac{Z_{I N}^{e}\left(l_{B}\right)}{Z_{0}} & \left(Z_{L B}^{e}-Z_{0}\right) \cdot \frac{T_{4}}{T_{3}} \\
& =\frac{Z_{I N}^{e}\left(l_{B}\right)}{Z_{0}}\left(Z_{L B}^{e}+Z_{0}\right),
\end{aligned}
$$$$
\left(Z_{L B}^{o}+Z_{0}\right) \cdot \frac{T_{1}}{T_{3}}+\left(Z_{L B}^{o}-Z_{0}\right) \cdot \frac{T_{2}}{T_{3}}-\frac{Z_{I N}^{o}\left(l_{B}\right)}{Z_{0}}\left(Z_{L B}^{o}-Z_{0}\right) \cdot \frac{T_{4}}{T_{3}}
$$

$$
=\frac{Z_{I N}^{o}\left(l_{B}\right)}{Z_{0}}\left(Z_{L B}^{o}+Z_{0}\right)
$$

where $Z_{L A}^{e}, Z_{L A}^{o}, Z_{L B}^{e}$, and $Z_{L B}^{o}$ are defined as a function of known parameters: $l_{A}, l_{B}$, and $\gamma$, and $Z_{I N}^{e}, Z_{I N}^{o}$ are known from the measured $S$ parameters of the lines $A$ and $B$ through Eqs. (11) and (12). Solving the system of Eq. (16) for $\left(T_{1} / T_{3}\right),\left(T_{2} / T_{3}\right),\left(T_{4} /\right.$ $\left.T_{3}\right)$, and using the reciprocity property of the transition $(\Delta=$ $\left.T_{T 11} \cdot T_{T 22}-T_{T 12} \cdot T_{T 21}=1\right), T_{3}$ can be obtained from

$$
\frac{1}{2} \cdot\left(\frac{T_{2}}{T_{3}}-\frac{T_{1}}{T_{3}} \cdot \frac{T_{4}}{T_{3}}\right)=\frac{1}{\left(T_{3}\right)^{2}}
$$

Once $T_{3}$ is calculated, $T_{1}, T_{2}$, and $T_{3}$ are computed from $\left(T_{1} / T_{3}\right)$, $\left(T_{2} / T_{3}\right)$, and $\left(T_{4} / T_{3}\right) ; T_{T 11}, T_{T 12}, T_{T 21}$, and $T_{T 22}$ are computed from Eq. (15), and the adapter $T$ matrix is determined.

\section{SIMULATION OF THE TRANSITION USING AN ELECTROMAGNETIC SIMULATOR}

In order to compare the proposed method to characterize the CPW-M transition, described in section 2, with the electromagnetic simulations (as suggested by other authors [11]), the 2.5D electromagnetic simulator Momentum (from Agilent) is used here. The simulation details are shown in Figure 3: the layout of a single CPW-M transition [Fig. 3(a)], and the transition layout dimensions [Fig. 3(b)]. The parameters of the alumina substrate used in the Momentum simulation are as follows: permittivity $\varepsilon_{r}=9.9$, metal thickness $t=3 \mu \mathrm{m}$, dielectric thickness $h=10 \mathrm{mil}$, conductivity $\sigma=4 \cdot 10^{7} \mathrm{~S} / \mathrm{m}$, loss tangent $\tan \delta=0.04$.

Prior to the transition simulation, Momentum is used to compute the $S$ parameters of a zero-length line, which is composed of two CPW-M transitions (see Fig. 1(a), "thru" connection, $l=0$ ) 


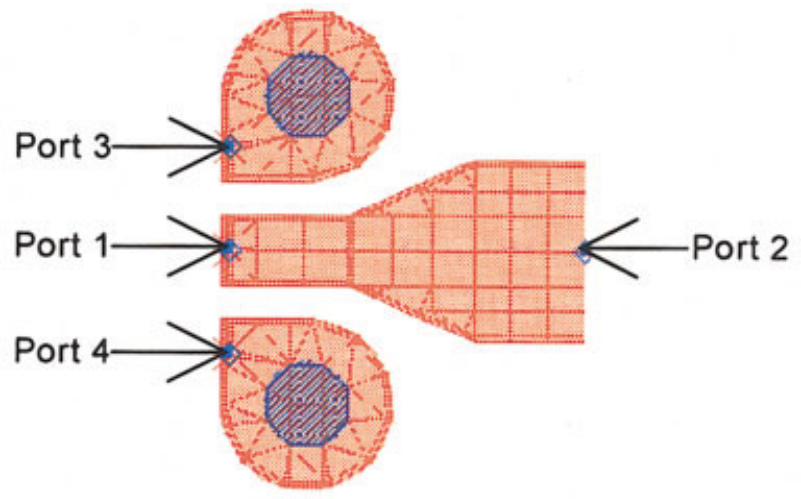

(a)

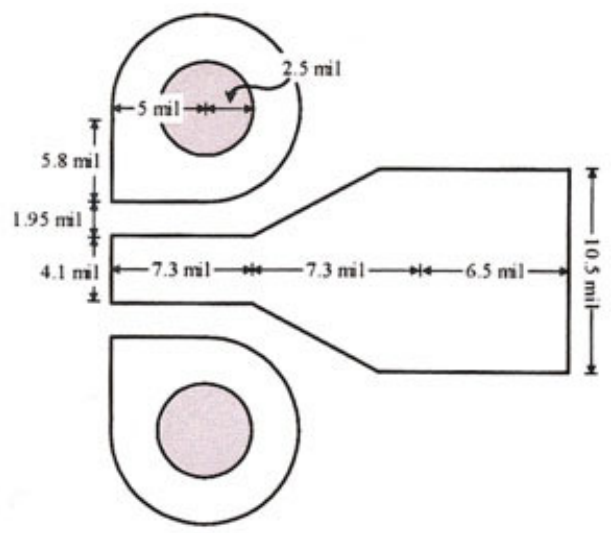

(b)

Figure 3 Layout used to simulate (a) the CPW-M transition and (b) the transition layout dimensions. [Color figure can be viewed in the online issue, which is available at www.interscience.wiley.com.]

and they are compared to the measured $S$ parameters. This calculation is developed to determine the loss tangent $\tan \delta$, since it is unknown, and the losses (return 0 insertion loss) are mainly due to this parameter. Once the loss tangent is known, the transition is simulated with Momentum using the half layout of a zero-length line [Fig. 3(a)]. The structure is defined as microstrip, therefore, via holes can be easily included. To consider the coplanar probe configuration, ground-signal-ground (G-S-G), three Momentum ports, at the probe position, are defined. The signal port [port 1 in Fig. 3(a)] is defined as an "internal port," the CPW ground ports [ports 3 and 4 in Fig. 3(a)] are defined as "ground" and associated with the internal port 1 . In this way, port 1 (CPW signal) is referred to the ground ports (CPW ground). The results obtained using the Momentum simulator are included into the dataset of a schematic circuit, the ground ports are connected externally to ground, and the internal port is connected to the reference load. In order to simulate the transition as a two-port one, a fourth port is connected to the microstrip-end as a single port [port 2 in Fig. 3(a)]. The results of the electromagnetic simulation and comparison with the experimental results are given in next section.

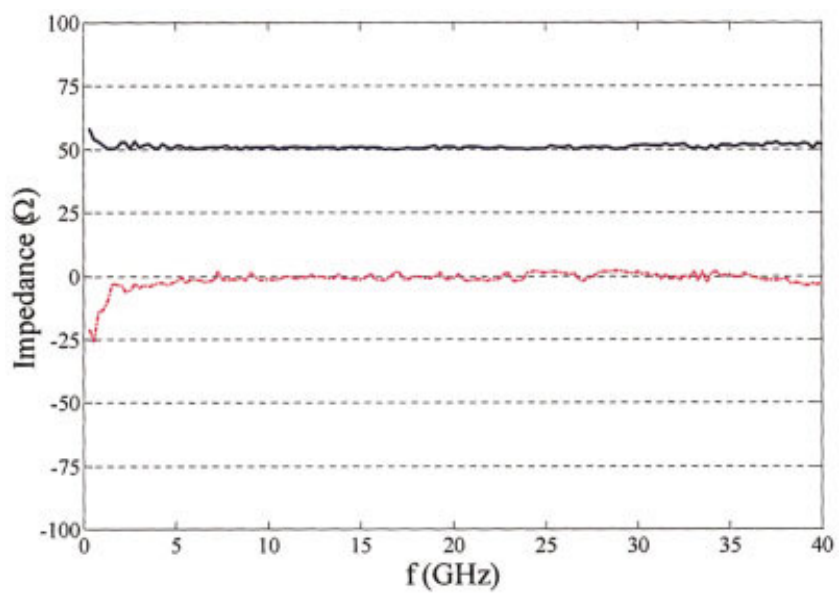

Figure 4 Extracted characteristic impedance of the microstrip transmission lines connected to CPW-M transitions: real part (-) and (- -) imaginary part. [Color figure can be viewed in the online issue, which is available at www.interscience.wiley.com.]

\section{EXPERIMENTAL RESULTS}

The method proposed in section 2 has been used to characterize a CPW-M transition from JMICRO ${ }^{\text {TM }}$ Technology. The $S$ parameters of two lines ( $A$ and $B$ ), each of which includes two CPW-M transitions, were measured. The length of line $A$ is $l_{A}=663 \mu \mathrm{m}$ (corresponding to an electrical delay of $5 \mathrm{ps}$ ), and the length of line $B$ is $l_{B}=1887 \mu \mathrm{m}$ (electrical delay of $15 \mathrm{ps}$ ). The measurement of the line $S$ parameters was made using an HP 8510C Automatic Network Analyzer connected to an on-wafer Probe Station (SUMMIT-9000) from Cascade-Microtech, equipped with ACP40-GSG150 cascade microprobes. On-wafer calibration was performed by applying the line-reflect-reflect-match (LRRM) method [8] to CPW (on-wafer) standards (Cascade-Microtech 101-190 LRM impedance standard substrate).

Figure 4 shows the line characteristic impedance obtained from Eq. (7). Its real part is very close to $50 \Omega$, and its imaginary part is negligible, except for the lower frequency range, where the real part increases and the imaginary part increases (negatively). This behavior is well known from transmission-line theory. Therefore, for transition characterization purposes, we can assume $Z \approx 50 \Omega$
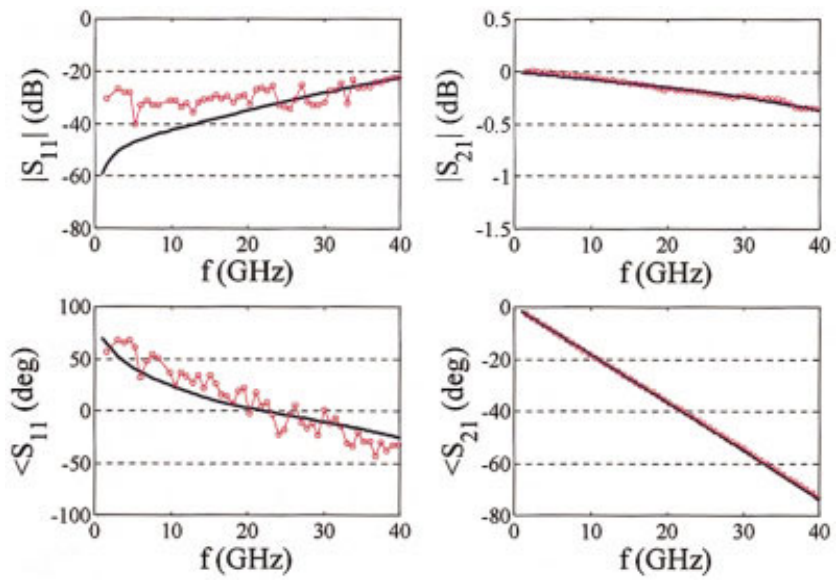

Figure 5 CPW-M transition extracted applying the proposed extraction method (-o-) and simulated with Momentum (-). [Color figure can be viewed in the online issue, which is available at www.interscience.wiley. com.] 

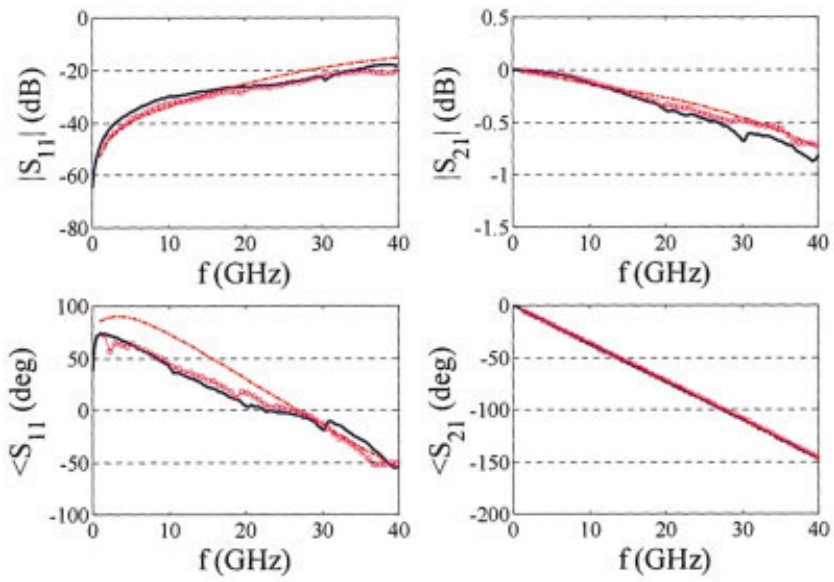

Figure 6 Comparison of the $S$ parameters of a zero-length line (0-ps thru line), measured (-), extracted with the proposed extraction method (-o-), and simulated with Momentum (- -). [Color figure can be viewed in the online issue, which is available at www.interscience.wiley.com.]

without significant variations in the adapter $S$ parameters' extraction.

Figure 5 shows the $S$ parameters, as a function of frequency, of the CPW-M transition computed from the $T$ matrix obtained from Eqs. (15)-(17), and those obtained from the Momentum electromagnetic simulation. The frequency response of $S_{11}$, estimated using the proposed method, is close to the Momentum simulation. The results for $S_{21}$ show very good agreement between the extraction method and Momentum simulation, for both magnitude and phase. To validate the proposed method to characterize CPW-M transitions, the $S$ parameters of a 0 -ps thru line [zerolength line, $l=0$ in Fig. 1(a)], which has not been used before for characterization, are calculated using the transition $T$ matrices $T_{T 1}$ and $T_{T 2}$, shown in Eq. (9). Then, the $T$ matrix of the thru line, $T_{T 1}$. $T_{T 2}$, is transformed into $S$ parameters. Also, the $S$ parameters of the thru line are simulated using Momentum. Figure 6 shows a comparison between directly measured thru-line $S$ parameters (using LRRM on-wafer calibration [8]), the $S$ parameters computed using the extracted transition $T$ matrix, and those simulated with Momentum. Very good agreement is observed between the results from transition extraction and experimental results, which validates the technique proposed herein.

As an example of the application of the proposed method to the measurement of microstrip devices, Figure 7 shows a coupled-line filter wire-bonded to two CPW-M transitions, with a center frequency $f_{0}=30 \mathrm{GHz}$ and bandwidth of $13 \%$. The filter $S$ parameters are measured at the coplanar plane with the same on-wafer probes and calibration mentioned above. Then, the filter $T$ param-

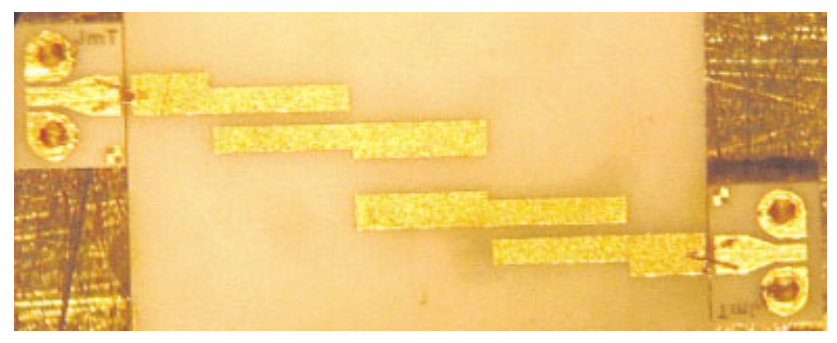

Figure 7 Coupled line filter wire-bonded to CPW-M transition. [Color figure can be viewed in the online issue, which is available at www. interscience.wiley.com.]
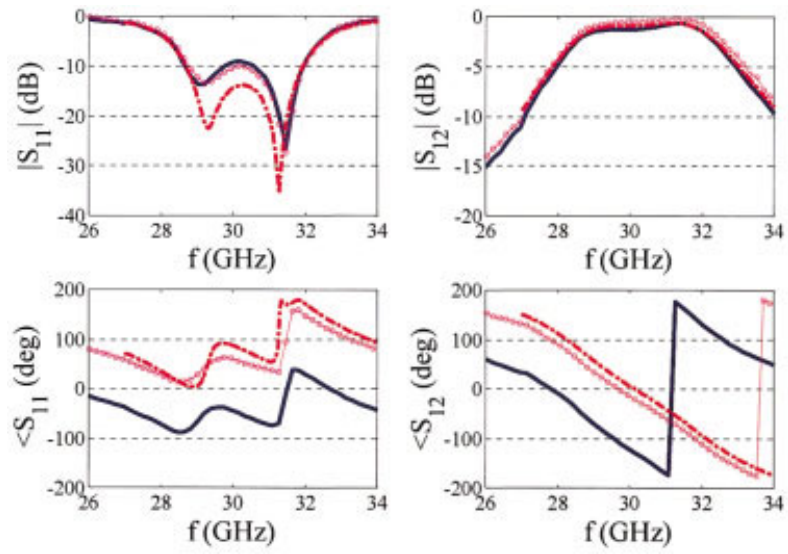

Figure $8 S$ parameters of a coupled line filter: measured at the coplanar plane (-), de-embedded at the microstrip plane (-o-), and simulated with Momentum, including the bonding wires (-.-). [Color figure can be viewed in the online issue, which is available at www.interscience.wiley.com.]

eters $T_{D U T M}$ at the microstrip plane are de-embedded using the following transition $T$ parameters:

$$
T_{D U T_{M}}=\left(T_{T 1}\right)^{-1} \cdot T_{D U T_{C P W}} \cdot\left(T_{T 2}\right)^{-1},
$$

where $T_{D U T C P W}$ is computed from the $S$ parameters measured at the CPW plane. Figure 8 plots the $S$ parameters measured at the CPW plane and de-embedded at the microstrip plane, and these are compared to those simulated with Momentum. The magnitude of the de-embedded $S_{11}$ shows a similar frequency response as the $S_{11}$ measured at the CPW plane, since the CPW-M transition and the coupled line filter are well-matched. However, the magnitude of the de-embedded $S_{12}$ is lower than those directly measured at the CPW plane because the CPW-M insertion loss contribution has been discounted. In the same way, a shift in the phases of $S_{11}$ and $S_{12}$ can be seen between the measurements at CPW and microstrip plane. The de-embedded $S$ parameters show good agreement with those obtained from the Momentum simulation.

\section{CONCLUSION}

In this paper a procedure for characterizing coplanar-to-microstrip (CPW-M) transitions has been presented. The transmission matrix ( $T$ matrix) of the CPW-M transition was extracted from the measured $S$ parameters of two microstrip lines, each of which include two CPW-M transitions. The results of the transition $T$-matrix extraction were compared to those obtained with electromagnetic simulations (using the Momentum simulator) and very good agreement was shown. To verify the results, the $S$ parameters of a thru line (zero-length line) were calculated using the extracted transition $T$ matrix and compared to those simulated with Momentum and with the $S$ parameters measured at the CPW planes. Excellent agreement among the $S$-parameters extracted, simulated, and measured was obtained. As an example of the application of CPW-M transitions to measure microstrip devices, the $S$ parameters of a two-port device (microstrip-coupled line filter), connected to two CPW-M transitions, were de-embedded at the microstrip plane from on-wafer measurements using the extracted $T$ matrix of the CPW-M transition. This technique can be generalized to other types of transitions such as coaxial-to-microstrip.

\section{ACKNOWLEDGMENTS}

This work has been supported by the Spanish Government under grants TIC2000-0144-P4-02 and ESP2002-04141-C03-02 (MCYT), and a scholarship from CONACYT-Mexico. 


\section{REFERENCES}

1. G.P. Gauthier, L.P. Katehi, and G.M. Rebeiz, W-band finite ground coplanar waveguide (FGCPW) to Microstrip line transition, IEEE MTT-S Dig, 1998, pp. 107-109.

2. T.J. Ellis, J.P. Raskin, L.P. Katehi, and G.M. Rebeiz, A wideband CPW-to-microstrip transition for millimeter-wave packaging, IEEE MTT-S Dig, 1999, pp. 629-632.

3. A.M.E. Safwat, K.A. Zaki, W. Johnson, and C.H. Lee, Novel design for coplanar waveguide to microstrip transition, IEEE MTT-S Dig, 2001, pp. 607-610.

4. G.F. Engen and C.A. Hoer, Thru-reflect-line: An improved technique for calibrating the dual six port automatic network analyzer, IEEE Trans Microwave Theory Tech 27 (1979), 987-993.

5. J.P. Mondal and T.-H. Chen, Propagation constant determination in microwave fixture de-embedding procedure, IEEE Trans Microwave Theory Tech 36 (1988), 706-713.

6. R.B. Marks, A multiline method of network analyzer calibration, IEEE Trans Microwave Theory Tech 39 (1991), 1205-1215.

7. H.-J. Eul and B. Shieck, Robust algorithms for TXX VNA selfcalibrations procedures, IEEE Trans Instrumentation and Measurement 43 (1994), 18-23.

8. F. Purroy and L. Pradell, New theoretical analysis of the LRRM calibration technique for vector network analyzers, IEEE Trans Instrumentation and Measurement 50 (2001), 1307-1314.

9. C. Wan, B. Nauwelaers, and W.D. Raedt, A simple error correction method for two-port transmission parameter measurement, IEEE Microwave Guided Wave Lett 8 (1998), 58-59.

10. A. Pham, J. Laskar, and J. Schappacher, Development of on-wafer microstrip characterization techniques, ARFTG Conf, 1997, pp. 8594.

11. W. Wiatr, Coplanar-waveguide-to-microstrip transition model, IEEE MTT-S Dig, 2000, pp. 1797-1800.

12. A.C. Ng, L.H. Chua, G.I. Ng, H. Wang, J. Zhou, and H. Nakamura, Broadband characterisation of CPW transition and transmission line parameters for small reflection up to $94 \mathrm{GHz}$, Asia-Pacific Microwave Conf, 2000, pp. 311-315.

13. J. Grzyb and G. Tröster, Characteristic impedance de-embedding of printed lines with the probe-tips calibration, $32^{\text {nd }}$ Euro Microwave Conf Proc, 2002, pp. 1069-1072.

(C) 2003 Wiley Periodicals, Inc.

\section{AN ITERATIVE METHOD FOR SOLVING A LARGE DENSE MATRIX IN THE METHOD OF MOMENTS SOLUTION OF AN ELECTROSTATIC PROBLEM}

Ritu Singh and Surendra Singh

Department of Electrical Engineering

The University of Tulsa

Tulsa, Oklahoma 74104

Received 8 May 2003

ABSTRACT: This paper illustrates the application of Wynn's vector $\varepsilon$-algorithm to solve a system of equations arising in the method of moments (MoM) solution of an electrostatic problem. Since the method is iterative, it does not require inversion of a matrix. The degree of accuracy of the solution can be controlled by specifying a convergence factor. (C) 2003 Wiley Periodicals, Inc. Microwave Opt Technol Lett 39: 378-380, 2003; Published online in Wiley InterScience (www.interscience.wiley.com). DOI 10.1002/mop. 11223

Key words: integral equations; method of moments; iterative method; solution of dense matrix; Wynn's vector algorithm

\section{INTRODUCTION}

Obtaining the numerical solution of electromagnetic radiation or scattering problems by using the method of moments (MoM) leads to a system of linear equations, which can be solved by matrix inversion, Gaussian elimination, or iterative techniques. However, an inherent limitation of the MoM is that it results in the generation of a dense matrix, which makes the solution process very time consuming, specifically, for electrically large structures. To overcome this limitation and to extend the application of MoM to the high-frequency regime, several alternative techniques have been suggested. The methods suggested so far include the multilevel fast-multiple algorithm [1], the impedance-matrix localization [2], the application of specially constructed adaptive-basis functions [3], and iterative methods such as the conjugate-gradient method [4] and the method of steepest descent [5].

In this work, we present an iterative technique for solving the MoM matrix problem. The method is based on the vector $\varepsilon$-algorithm proposed by Wynn [6]. The application of the method is illustrated by applying it to find the charge distribution on a conducting strip using the MoM. Since this method is iterative, matrix inversion is not needed. The solution process can be stopped when a predefined convergence criterion is met. This is advantageous, as higher accuracy in the solution can be obtained by making the convergence criterion more stringent. The storage requirements needed in the solution process are considerably modest. Typically, to solve an $N \times N$ matrix problem, the method requires a working space equivalent to two $N \times p$ matrices where $p \ll N$. The numerical results presented here illustrate the usefulness of the method. The advantage of Wynn's vector $\varepsilon$-algorithm over the biconjugate gradient method has been pointed out by Brezinski [7]. The biconjugate-gradient method's limitation is that it may have a breakdown, which would cause the algorithm to stop, or a near breakdown, which would cause the propagation of round-off errors, resulting in a failure of convergence. In Wynn's algorithm, such a breakdown or near breakdowns are curable. Brezinski has shown that under certain conditions, Wynn's algorithm can be used to solve a system of linear equations, $A X=b$, where $A$ is a real, singular, square matrix [6].

\section{WYNN'S VECTOR $\varepsilon$-ALGORITHM}

Let us solve, say, the following matrix problem given by the system of linear equations:

$$
A X=b \text {, }
$$

where $A$ is a known $N \times N$ MoM matrix $A=\left[a_{i j}\right], X$ is an unknown $N \times 1$ column vector, $X=\left(x_{1}, x_{2}, \ldots, x_{N}\right)^{T}$, and $b$ is a known $N \times 1$ forcing function vector $b=\left(b_{1}, b_{2}, \ldots, b_{N}\right)^{T}$. Wynn's algorithm provides an estimate of $X$ without the use of matrix inversion.

In the $\varepsilon$-algorithm, the starting vectors $\left\{\varepsilon_{-1}^{(0)}\right\}$ and $\left\{\varepsilon_{0}^{(q)}\right\}$, each of length $N$, are constructed from the initial values:

$$
\begin{aligned}
& \left\{\varepsilon_{-1}^{(q)}\right\}=\{0\}, \quad q=1,2, \ldots \\
& \left\{\varepsilon_{0}^{(0)}\right\}=\left\{S^{(0)}\right\}=\{0\}, \\
& \left\{\varepsilon_{0}^{(q)}\right\}=\left\{S^{(q)}\right\}, \quad q=1,2, \ldots
\end{aligned}
$$

The initial vectors, $S^{(q)}=\left(s_{1}^{(q)}, s_{2}^{(q)}, \ldots, s_{N}^{(q)}\right)$, can be calculated using the Gauss-Seidel relaxation [8]: 